\title{
COMPARISON OF A MOSAIC-CRYSTAL SPECTROMETER TO A HIGH-PERFORMANCE SOLID-STATE DETECTOR FOR X-RAY MICROFLUORESCENCE ANALYSIS \\ CONF- $980405-$
}

J.-S. CHUNG*, S. ISA*, C. J. SPARKS*, G. E. ICE*, S. McHUGO** and A. THOMPSON**

*Oak Ridge National Laboratory, P.O. Box 2008, Oak Ridge TN 37830

**Lawrence Berkeley National Laboratory, 1 Cyclotron Rd., Berkeley CA 94720

\section{ABSTRACT}

RECEIVEO JUN 249998

0 QTI

- The minimum-detectable-limit of a compact double-focusing graphite mosaic-crystal spectrometer is compared to the minimum-detectable-limit from a high-performance Ge solidstate detector. The solid angle and efficiency of the solid-state detector is much greater than for the crystal spectrometer. However, the better signal-to-noise of the spectrometer and its insensitivity to matrix fluorescence and scattering can give it a better minimum-detectable-limit for trace element analysis. The relative advantages of the two detectors are illustrated for some simple test samples. The performance of the crystal spectrometer compared to the solid-state detector increases as the flux in the $\mathrm{x}$-ray probe increases. This makes crystal spectrometers especially interesting for use with new high intensity 3 rd generation synchrotron microprobes. An estimate is made of the source and sample conditions favored for each detector.

\section{INTRODUCTION}

$\mathrm{X}$-ray fluorescence analysis with small electron, proton or $\mathrm{X}$-ray beams is widely used for the identification of elemental distributions. ${ }^{1-3}$ The figure-of-merit for fluorescence analysis is the minimum-detectable-limit: literally the minimum concentration which can generate a significant signal above background. Minimum detectable limit (MDL) depends on the intensity and nature of the excitation probe, the efficiency of the detector, the data collection time, the detector bandpass, and the sample composition or "matrix". We adopt the definition of minimum detectable limit given by Sparks $s^{4}$. The MDL can be determined from signal and background of a known standard,

$$
C_{M D L}=3.29 C_{Z} \frac{\sqrt{N_{b}}}{N_{s}} .
$$

Here $C_{M D L}$ is the minimum detectable limit, $C_{Z}$ is the mass fraction in a measured standard. $N_{b}$ is the background counts beneath the standard fluorescence signal and $\mathrm{N}_{\mathrm{S}}$ is the net counts at the fluorescenceenergy.

$\mathrm{X}$-ray excitation typically creates a much smaller background than charged particle probes (Ref 4) and monochromatic $x$-ray sources have the best signal-to-noise. Until recently however. monochromatic $\mathrm{x}$-ray beams with $\mu \mathrm{m}^{2}$ cross sections have been very weak. The advent of intense 3 rd generation synchrotron sources however, has now stimulated renewed interest in $\mathrm{x}$-ray excited microfluorescence analysis. ${ }^{5.6}$

To efficiently use these powerful new sources it is important to match the detector to the source. Fluorescence spectra are typicallv measured with a solid-state detector ${ }^{7}$ although curved "The submitted manuscript has been authored by a contractor of the U.S. Government under contract No. DE-AC05960R22464. Accordingly, the U.S. Government retains a nonexclusive, royalty-free license to publish or reproduce the published form of this contribution. or allow others to do so. for U.S. Government purposes." 


\section{DISCLAIMER}

This report was prepared as an account of work sponsored by an agency of the United States Government. Neither the United States Government nor any agency thereof, nor any of their employees, makes any warranty, express or implied, or assumes any legal liability or responsibility for the accuracy, completeness, or usefulness of any information, apparatus, product, or process disclosed, or represents that its use would not infringe privately owned rights. Reference herein to any specific commercial product, process, or service by trade name, trademark, manufacturer, or otherwise does not necessarily constitute or imply its endorsement, recommendation, or favoring by the United States Government or any agency thereof. The views and opinions of authors expressed herein do not necessarily state or reflect those of the United States Government or any agency thereof. 
and mosaic crystal analyzers ${ }^{8,9}$ are also used. In addition to the natural background generated in the sample, a solid-state detector contributes background due to insufficient charge collection. A solid-state detector also has (in general) poorer energy resolution than a crystal spectrometer and its count-rate limit is set by the major elements in the sample matrix rather than by the trace elements. However a solid-state detector has a large advantage in terms of total solid angle and efficiency compared to a crystal spectrometer. A solid-state detector also simultaneously measures all elements in the sample matrix with $Z>18$.

Here we compare the MDL which can be achieved with a high-performance Ge solid state detectors to the MDL achievable with a double-focusing mosaic-graphite crystal spectrometer. The aim of this paper is to define the experimental conditions which favor the two detectors.

\section{EXPERIMENTAL SETUP}

\section{Source and geometry}

Measurements were made on beamline 10.3.1 at the Advanced Light Source (ALS). This beamline utilizes graded elliptical Kirkpatrick-Baez $(\mathrm{KB})$ multilayers to focus the beam. The multilayer optics reflect an $\sim 6 \%$ bandpass from the incident white synchrotron radiation beam. Selectable mirrors allow several bandpass options. For the present measurements the multilayer mirrors were an $\sim 12.5 \mathrm{keV}$ pair which focused $\sim 10^{9}$ photons into $\sim 1 \times 1.5 \mu \mathrm{m}^{2}$. For white beam measurements the upstream slits of the monochromator tank were used to collimate the white beam to $\sim 50 \times 250 \mu \mathrm{m}^{2}$. The intensity of Fe fluorescence excitation was $\sim 200 \mathrm{x}$ greater with the collimated white beam than with the focused beam. Hence the flux density in the focused spot was $\sim 50 \mathrm{x}$ greater for the focused beam even though it had only a $4 \%$ bandpass.

The detector was placed perpendicular to the incident $x$-ray beam in the plane of the ring. The sample was placed in the bisecting geometry. This orientation minimized elastic and Compton scattering. Measurements were made with both focused monochromatic x-rays ( $4 \%$ bandpass) and with collimated white beams.

\section{$\underline{\text { Samples }}$}

Two standards were used to measure the MDL over a range of experimental conditions. A sample with $0.4 \mathrm{Wt}$. \% Fe in an $\mathrm{Al}$ matrix was prepared to study the case of a trace impurity in a low $\mathrm{Z}$ matrix. Al and. Fe powder were mixed, then pressed into a pellet. A powder pellet formed from ultra-pure $\mathrm{Al}(99.999 \%)$ was also made as a background standard. For the case of a trace impurity in a high $\mathrm{Z}$ matrix, a sample of $\mathrm{Fe}$ in $\mathrm{W}$ was prepared. $\mathrm{Fe}_{2} \mathrm{O}_{3}$ powder and $\mathrm{WO}_{3}$ powders were mixed together in the mass fractions which made $1 \mathrm{Wt}$. \% of $\mathrm{Fe}$ in total mass. A pure $\mathrm{WO}_{3}$ pellet was made for a background standard.

\section{$\underline{\text { Detectors }}$}

Ge Solid State Detector. The solid-state (energy dispersive) detector was a single-element high-purity Germanium detector from EG\&G ORTEC (Model No. IGLET-11145S). This detector incorporates an ultra-thin detector entrance window which allows for good collection efficiency for low $Z$ elements and high peak-to-background ratios. The preamplifier electronics are based on a pulsed reset circuit which allows for high count rate. For Fe 55 radiation with an $\mathrm{Mn} \mathrm{K}_{\alpha}$ fluorescence energy of $5.9 \mathrm{keV}$, the IGLET detector has a resolution of $137 \mathrm{eV}$ with a 10 $\mu$ s amplifier shaping time and a $110 \mathrm{~mm}^{2}$ active area. With an active area of $110 \mathrm{~mm}^{2}$ the solid angle which can be accepted by this detector is $\sim 110 / \mathrm{d}^{2}(\mathrm{~mm})$. 
Solid-state detectors have a trade-off between count rate and energy resolution. With a $10 \mu \mathrm{s}$ shaping constant, the effective detector deadtime is $\sim 30 \mu \mathrm{s}$. For a non-paralyzable detector this results in an $\sim 10 \%$ deadtime at 3,000 cps. The amplifier shaping constant can be reduced to improve the count rate, but only at the expense of energy resolution. For example, at $1 \mu \mathrm{s}$ shaping constant a $10 \%$ deadtime will not occur until $30,000 \mathrm{cps}$. With a $1 \mu \mathrm{s}$ amplifier shaping constant however the energy resolution degrades by a factor of $\sim 2$ at $5.9 \mathrm{keV}$. From Eq. 1 it is clear that higher count rate is favored over good energy resolution for an isolated fluorescence line. For example, if background scales linearly with energy resolution, a $1 \mu$ s shaping constant compared to a $10 \mu$ s shaping constant, will allow a factor of 10 high count rate with only a factor of 2 higher background. This should yield an $\sim 2.2$ times smaller MDL assuming the solid-angle or probe intensity can be scaled to maintain the count-rate at $\sim 10 \%$ deadtime.

In actual trace-element experiments however the background can often increase much faster than linearly with energy resolution. This is especially true if the spectra near the characteristic line of interest contains an intense peak. Furthermore, the total deadtime of a solid state detector depends on the integrated deadtime over the whole spectrum and not on the count rate at the fluorescence line of interest. This means that the count-rate of a trace-element measurement with a solid-state detector is limited by fluorescence from all of the elements in the sample matrix rather than from the trace elements of interest.

One approach to the problem of matrix fluorescence is to use a graphite prefilter to a solid state detector. With large $\left(\sim 10^{4} \mu \mathrm{m}^{2}\right)$ beams and long $\left(\sim 10^{3} \mathrm{~s}\right)$ counting times this approach has been successfully used to study heavy metals in polymers and toxic trace metals with 10-100 PPB sensitivity. ${ }^{10,11}$ Another interesting approach uses a Kumakov lens to collimate the X-ray beam before energy analysis with a mosaic crystal. ${ }^{12}$ Best signal-to-noise and lowest MDL (for non count-rate limited measurements) however is realized with a focusing wavelength-dispersivespectrometer (WDS) of which our mosaic crystal spectrometer is one example. ${ }^{9.12}$

Mosaic-Crystal Spectrometer. The mosaic crystal (wavelength dispersive) spectrometer is a compact version of a mosaic-crystal spectrometer described in a previous paper. ${ }^{9}$ A schematic of the spectrometer is shown in Fig. 1. The basic elements of the spectrometer are a monochromator housing, a curved graphite monochromator crystal, and a compact linear position sensitive detector. The curved graphite crystal had a sagittal (out of the diffracting plane) curvature of $\sim 29 \mathrm{~mm}$, and a mosaic spread of $\sim 0.8$ degrees. X-rays are parafocused onto the detector in a 1:1 magnification geometry. The condition for optimum sagittal focusing is given by, ${ }^{9} F_{1}=R_{s} / \sin \left(\theta_{B}\right)$. Here $R_{S}$ is the sagittal radius. $F 1$ is the sample to crystal distance and $\theta_{B}$ is the Bragg angle. The useful solid angle of the detector is determined by the effective width and length of the mosaic crystal $\left(\sim 10 \times 35 \mathrm{~mm}^{2}\right)$, the distance to the sample, and the Bragg angle, $\sin \left(\theta_{B}\right)=1.8478 / E(\mathrm{kev})$. The effective solid angle of the mosaic crystal is reduced because of the $\sim 40 \%$ reflectivity of the mosaic crystal. The effective solid angle, $S_{E}$, of wavelength-dispersive spectrometer is,

$$
S_{E}=0.4 \times 350\left(\mathrm{~mm}^{2}\right) \frac{1.8478}{E(\mathrm{keV})}\left\{\frac{1.8478(\mathrm{~mm})}{29(\mathrm{~mm}) E(\mathrm{keV})}\right\}^{2}=\frac{1.05}{E(\mathrm{keV})^{3}}
$$

At $8 \mathrm{keV}$, the solid angle of the mosaic crystal detector is $\sim 2 \times 10^{-3}$ whereas the solid angle of the Ge detector at $d=50 \mathrm{~mm}$ from the sample is $\sim 4 \times 10^{-2}$ (20x higher).

The linear detector is a specially designed ORDELA model 1010X Xe linear proportional counter. This detector has a $1 \mathrm{~cm}$ long by $0.8 \mathrm{~cm}$ wide active area with a spatial resolution of $\sim 40$ $\mu \mathrm{m}$. The theoretical energy resolution of the wavelength-dispersive spectrometer (WDS) as a function of x-ray energy and x-ray probe size is calculated based on reference 9 (Fig. 2). The 
measured energy resolution was $\sim 46 \mathrm{eV}$ which is about $40 \%$ worse than anticipated.

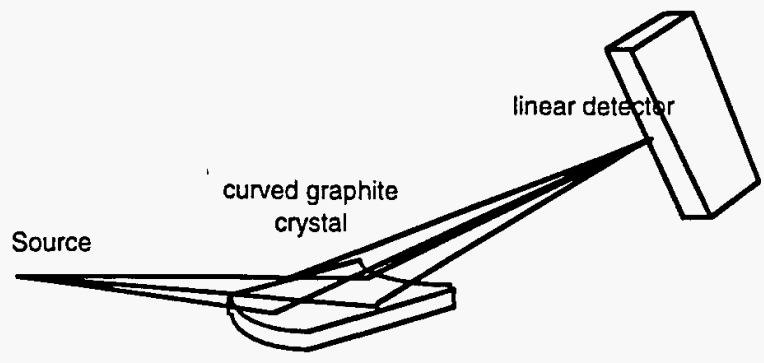

Fig. 1. Schematic of mosaic crystal spectrometer showing the essential components.

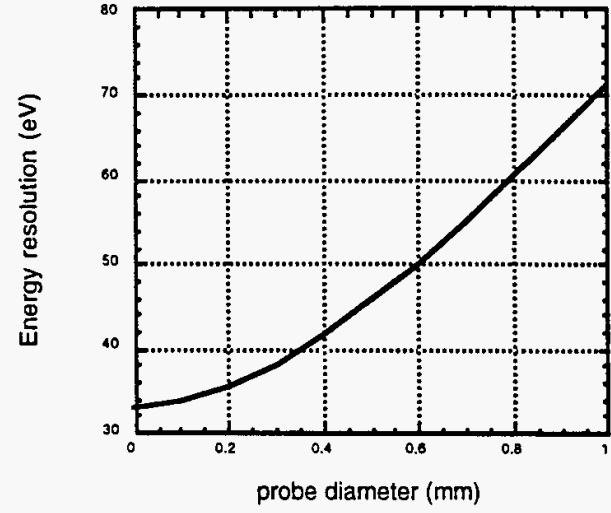

Fig. 2. Theoretical energy resolution at 6.4 $\mathrm{keV}$ as a function of source size.

\section{EXPERIMENTAL MEASUREMENTS}

The mosaic analyzer crystal was mounted $10.2 \mathrm{~cm}$ from the sample to optimize the focus and signal-to-noise. The Ge detector was moved as close as possible to the sample without exceeding $10 \%$ deadtime. An amplifier shaping constant of $10 \mu$ s was used. Data were collected and compared for the two detectors with both white (collimated) and monochromatic (focused) beams. With the Ge detector the lowest MDL was measured with the focused monochromatic beam; monochrmatic beams have lower intrinsic background and the detector was already count rate limited. With the WDS detector best MDL was achieved with the white beam (see description below). All spectra, background counts and fluorescence signals were compared in the region of $+/-3 \sigma$. Here $\sigma$ 's were determined by least square fittings to Gaussian curves.

\section{Crystal Spectrometer}

Fig. 3 illustrates the performance of the WDS. The bottom of Fig. 3 superimposes the measured spectra with Fe impurity on the spectra measured off ultra pure Al. As can be seen, the crystal spectrometer background analysis is complicated by the significant Lorentzian component of the $K \alpha$ line and by the non-uniform energy response of the spectrometer; the crystal spectrometer background exhibits a broad Gaussian shape arising from the increasing efficiency of the crystal spectrometer near the nominal center of its range. A $99.999 \%$ pure $\mathrm{Al}$ powder sample was used to measure the background. The background is dominated by a broad Gaussian which corresponds to elastic scattering within the 0.5 degree mosaic spread of the graphite crystal. As shown in Fig. 3 this background is much broader than the peak associated with $\mathrm{Fe} \mathrm{K}$ alpha radiation.

A further complication for this particular setup was the unfortunate overlap of $\mathrm{x}$-ray elastic scattering from the graphite $(00 \bullet 4)$ reflection at $\sim 12.8 \mathrm{keV}$ onto the detector. Because the energy bandpass of the multilayer mirrors was almost exactly twice the energy of the fluorescence line of interest, the monochromatic beam had an unusually high $\lambda / 2$ background. About $80 \%$ of this background was suppressed by gating the linear proportional counter sum pulse. However, the major signal-to-noise improvement expected for monochromatic beam as compared to white beam 
was not realized with this setup due to the $\lambda / 2$ contamination.

$\mathrm{Al}+\mathrm{Fe}$, White beam, Graphite mono.
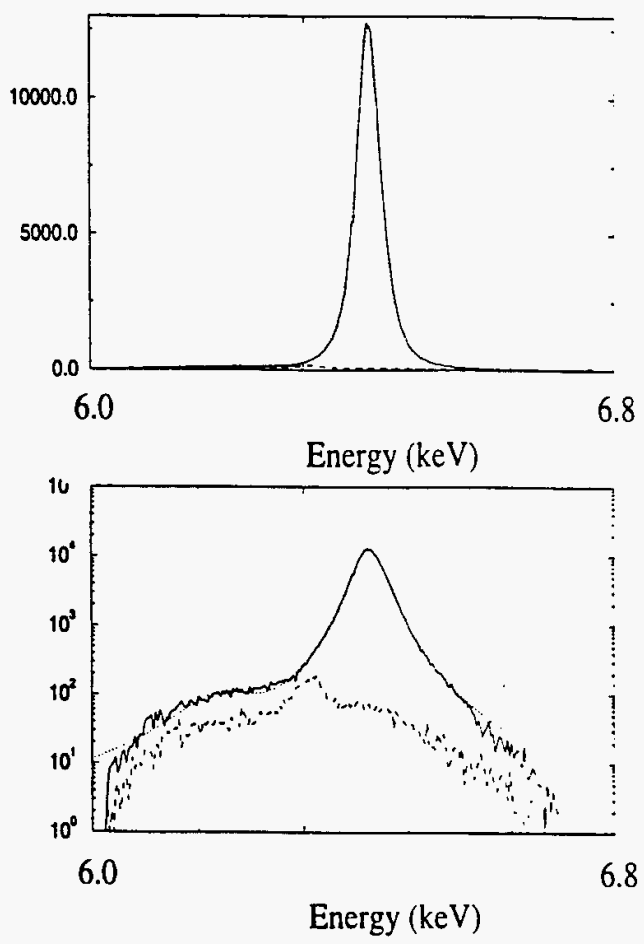

Fig. $3 \mathrm{Fe} \mathrm{K} \alpha$ line as measured by the graphite spectrometer. The top plot shows the $\mathrm{Fe} \mathrm{K} \alpha$ line on a linear scale. The bottom line superimposes the background from uitra pure $\mathrm{Al}$ on the measured spectra from the standard.
$\mathrm{Al}+\mathrm{Fe}, \mathrm{Ge}$ detector, monochromatic
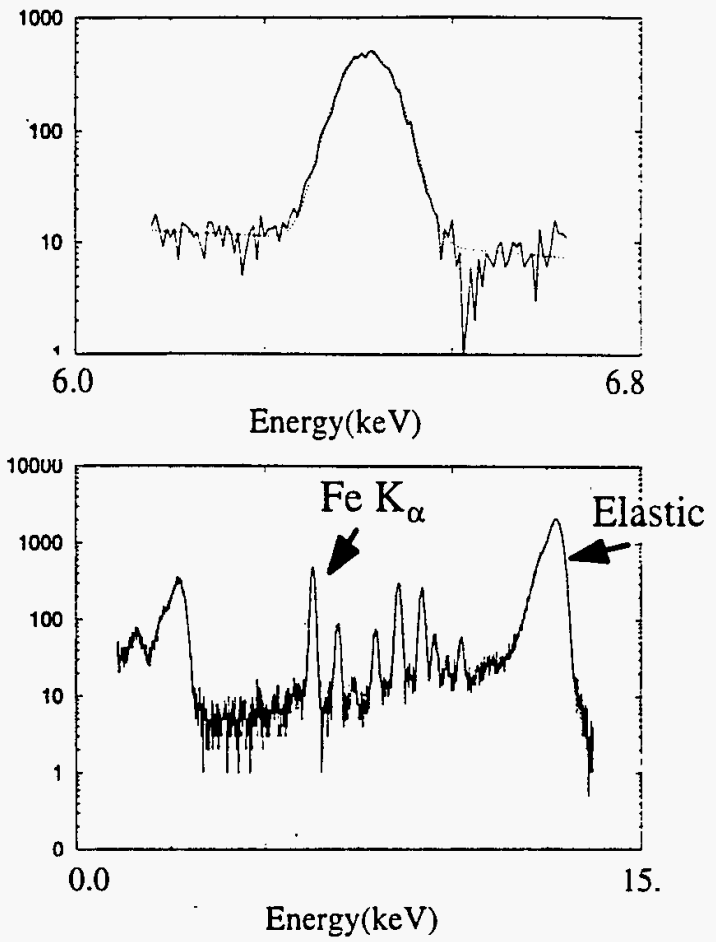

Fig. 4 Bottom figure shows the overall spectra measured by the Ge detector whilethe top figure shows an enlargement of the $\mathrm{Fe} \mathrm{K} \alpha$ region.

The mosaic-crystal spectrometer spectra off the $\mathrm{Fe}_{2} \mathrm{O}_{3}$ in a $\mathrm{WO}_{3}$ matrix is similar to the spectra observed with an $\mathrm{Al}$ matrix. However, the heavy $\mathrm{Z}$ matrix has lower signal-to-noise and hence ahigher minimum-detectable-limit.

\section{Ge Detector}

The spectra from the Fe-Al sample. measured with the Ge detector, is shown in Figure 4. This spectra was collected with a focused monochromatic beam with $\sim 200$ times less fluorescing power than the collimated white beam. Trace element count rates are limited primarily however by deadtime restrictions imposed by the elastic scattering peak in the $\mathrm{FeAl}$ sample. In the $\mathrm{Fe}_{2} \mathrm{O}_{3}$ $\mathrm{WO}_{3}$ sample, $\mathrm{W}$ fluorescence restricted the acceptable solid-angle of the detector. In addition, for the $\mathrm{Fe}_{2} \mathrm{O}_{3}$ sample, the $\mathrm{Fe} \mathrm{K} \alpha$ line lies on top of a sloping background from the $\mathrm{W}$ lines.

The count rates, signal-to-background and minimum detectable limits for the three samples are summarized in Table 1. As can be seen, because the count rate in the SSD is limited by the matrix signal, the WDS can achieve better MDL even with a white beam. Much better signal-to-noise is possible if a monochromatic $\mathrm{x}$-ray beam $\sim 10 \%$ above the absorption edge of the trace elements is used. The MDL for the solid-state detector however will remain virtually fixed with increased signal because it has already reached its deadtime limit. Improved MDL for the SSD will require multiplexing the detector. We note that $\sim 400$ solid state detectors are needed to match the MDL of the single mosaic crystal spectrometer. 
With the experimental setup used in this example, the MDL can be estimated as a function of beam intensity. We ratio the flux to $I_{O} \equiv$ flux in the focused monochromatic beam on $10.3 .1\left(\sim 10^{9}\right.$ $12.5 \mathrm{keV}$ x-rays). As can be seen the solid-state detector outperforms the crystal spectrometer at low flux due to its much higher collection efficiency. For high intensity $x$-ray beams however the deadtime limitations of the solid-state detector restrict the MDL which it can achieve. The estimated flux at the APS is $\sim 4$ orders of magnitude more than on beamline10.3.1.

The solid-state detector does particularly well compared to the crystal-spectrometer on systems with low $\mathrm{Z}$ matrices and for weak incident beams. It is also at a big advantage if more than one element must be monitored simultaneously.

Table 1. Summary of the performance of the two detectors. Counting time was 1000 seconds.

\begin{tabular}{|l|l|l|l|l|l|l|}
\hline & \multicolumn{3}{|c|}{$\begin{array}{c}\text { Mosaic-Crystal Spectrometer } \\
\text { (collimated white beam) }\end{array}$} & \multicolumn{3}{c|}{$\begin{array}{c}\text { Solid-state detector } \\
\text { (focused monochromatic beam) }\end{array}$} \\
\hline & Counts & $\begin{array}{l}\text { Signal-to- } \\
\text { noise }\end{array}$ & $\begin{array}{l}\text { MDL } \\
(\mathrm{ppm})\end{array}$ & Counts & $\begin{array}{l}\text { Signal-to- } \\
\text { noise }\end{array}$ & $\begin{array}{l}\text { MDL } \\
\text { (ppm) }\end{array}$ \\
\hline $\mathrm{Al}$ powder & 208,000 & $75: 1$ & 2.3 & 3750 & $14: 1$ & 40 \\
\hline $\mathrm{Fe}_{2} \mathrm{O}_{3}+\mathrm{WO}_{3}$ & 564,600 & $28: 1$ & 5.8 & 14200 & $2.6: 1$ & 121 \\
\hline
\end{tabular}

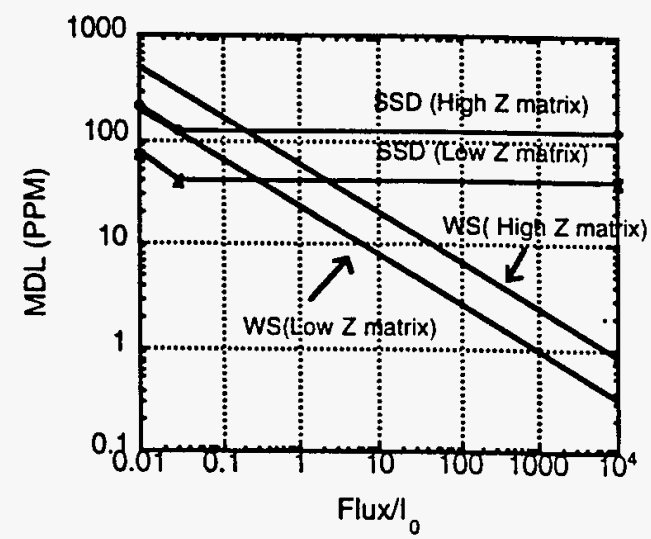

Fig. $6 \mathrm{MDL} / \mathrm{s}$ for an $\mathrm{x}$-ray microprobe as a function of flux. $\mathrm{I}_{0}$ is the nominal intensity of the focused $\mathrm{x}$-ray beam on ALS beamline 10.3.1 ( $\sim 10^{9}$ photons/s). Note the MDL in table 1 is for $1000 \mathrm{~s}$.

\section{CONCLUSIONS}

For some simple test samples, a compact mosaic crystal spectrometer has been shown to yield lower minimum-detectable-limits than an advanced solid-state detector. The relative advantages of the two detectors depends on the sample being measured and on the intensity of the source. For the most advanced $x$-ray microprobe beams, mosaic crystal spectrometers are favored because they are not paralyzed by the intense fluorescence of major matrix components of the sample, and because they have better signal-to-noise. 


\section{ACKNOWLEDGMENTS}

Research sponsored by the Laboratory Directed Research and Development Program of the Oak Ridge National Laboratory and by the Office of Basic Energy Sciences, Division of Materials Sciences, U.S. Department of Energy under contract DE-AC05-96OR22464 with Lockheed Martin Energy Research Corporation. Research performed in part at beamline 10.3.1 at the ALS which is sponsored by Basic Energy Sciences U.S. Department of Energy.

\section{REFERENCES}

1. J.R. Chen, E.C. T. Chao, J.A. Minkin, J. M. Back, K.W. Jones, M.L. Rivers and S.R. Sutton, Nucl. Instr. and Meth. B49 533 (1990).

2. D.R. Beaman and L.F. Solosky, Anal. Chem. 44 1598-1610 (1972).

3. G.J.F. Legge and A. Saint, Nucl. Inst. and Meth B49 418 (1990).

4. C.J. Sparks, "X-ray Fluorescence Microprobe for Chemical Analysis", in Synchrotron Radiation Research, edited by H. Winick and S. Doniach, 459 (Plenum Press 1980); L. A. Currie, Anal. Chem. 40587 (1968).

5. P. Chevallier and P. Dhez, Hard X-ray Microbeam: Production and Application in Accelerator based atomic physics techniques and applications (S.M. Shafroth and J. Austin eds.) pp. 309348 AlP Press, New York.

6. G.E. Ice, $X$-ray Spectrometry 26 315-326 (1997).

7. A.D. Smith, G.E. Derbyshire, R.C. Farrow, A. Sery, T.W. Raudorf and M. Martini, Rev. Sci. Instrum. 662333 (1995).

8. F. Folkmann and F. Frederiksen, Nucl. Inst. and Meth. B49 126 (1990).

9. G. E. Ice and C.J. Sparks, Nucl. Inst. and Meth. A291 110-116 (1990).

10. C.J. Sparks, L.A. Harris and O.B. Cavin, "Development of High Sensitivity X-ray Fluorescence for Analysis of Trace Toxic Elements", ORNL-NSF-EATC-1 (Progress Report)1972.

12. V. Baryshev, Y. Kolmogorov, G. Kulipanov, and A. Skrinsky, Nucl. Inst. and Meth A246 739 (1986).

13. J. P. Kirkland, V.E. Kovantsev, C.M. Dozier, J.V. Gilfrich, W.M. Gibson, Q.F. Xiao, K. Umezawa, Rev. Sci. Inst. 66 1410-1412 (1995). 

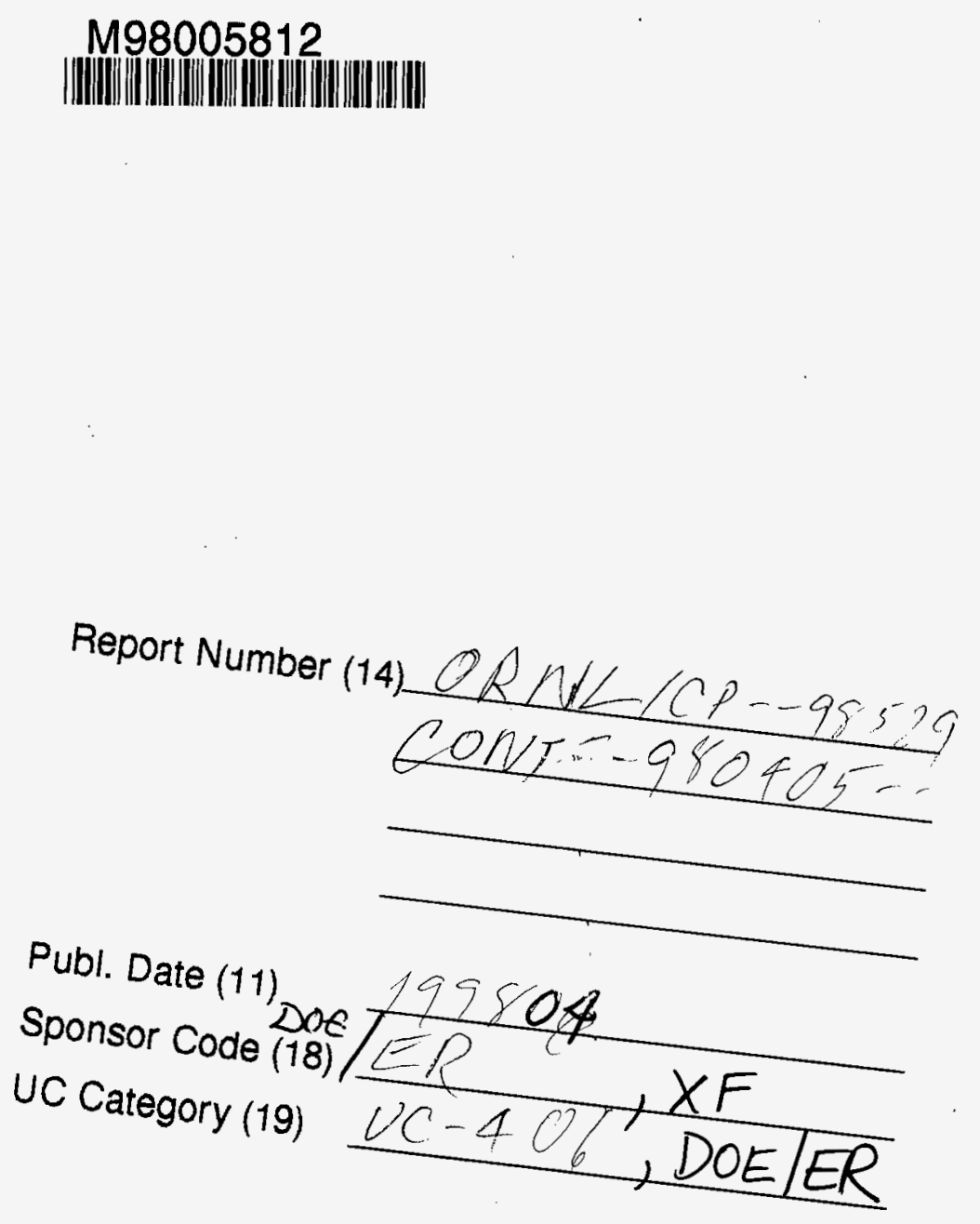

\section{9}

DTIC QUALITY INOPECTED 1 